\title{
A nonlocal multi-point multi-term fractional boundary value problem with Riemann-Liouville type integral boundary conditions involving two indices
}

\author{
Ahmed Alsaedi ${ }^{1}$, Sotiris K Ntouyas², Ravi P Agarwal ${ }^{1,3}$ and Bashir Ahmad ${ }^{1 *}$
}

"Correspondence:

bashirahmad_qau@yahoo.com 'Department of Mathematics,

Faculty of Science, King Abdulaziz University, P.O. Box 80203, Jeddah, 21589, Saudi Arabia

Full list of author information is available at the end of the article

\begin{abstract}
In this paper, we study the existence of solutions for fractional differential equations of arbitrary order with multi-point multi-term Riemann-Liouville type integral boundary conditions involving two indices. The Riemann-Liouville type integral boundary conditions considered in the problem address a more general situation in contrast to the case of a single index. Our results are based on standard fixed point theorems. Some illustrative examples are also presented.
\end{abstract}

MSC: 26A33; 34A08

Keywords: fractional differential equations; nonlocal boundary conditions; fixed point theorems

\section{Introduction}

In the last few decades, the subject of fractional differential equations has become a hot topic for the researchers due to its intensive development and applications in the field of physics, mechanics, chemistry, engineering, etc. For a reader interested in the systematic development of the topic, we refer the books [1-6]. A fractional-order differential operator distinguishes itself from the integer-order differential operator in the sense that it is nonlocal in nature, that is, the future state of a dynamical system or process involving fractional derivative depends on its current state as well its past states. In other words, differential equations of arbitrary order describe memory and hereditary properties of various materials and processes. As a matter of fact, this characteristic of fractional calculus makes the fractional-order models more realistic and practical than the classical integer-order models. There has been a great surge in developing the theoretical aspects such as periodicity, asymptotic behavior and numerical methods for fractional equations. For some recent work on the topic, see [7-25] and the references therein. In particular, the authors studied nonlinear fractional differential equations and inclusions of arbitrary order with multi-strip boundary conditions in [21], while a boundary value of nonlinear fractional differential equations of arbitrary order with Riemann-Liouville type multi-strip boundary conditions was investigated in [22]. Sudsutad and Tariboon [26] obtained some existence results for an integro-differential equation of fractional order $q \in(1,2]$ with $m$-point multi-term fractional-order integral boundary conditions.

○2013 Alsaedi et al: licensee Springer. This is an Open Access article distributed under the terms of the Creative Commons Attribution License (http://creativecommons.org/licenses/by/2.0), which permits unrestricted use, distribution, and reproduction in any medium, provided the original work is properly cited. 
In this paper, we study a boundary value problem of fractional differential equations of arbitrary order $q \in(n-1, n], n \geq 2$ with $m$-point multi-term Riemann-Liouville type integral boundary conditions involving two indices given by

$$
\left\{\begin{array}{l}
{ }^{c} D^{q} x(t)=f(t, x(t)), \quad t \in[0, T], \\
x(0)=0, x^{\prime}(0)=0, \ldots, x^{(n-2)}(0)=0, \quad x(T)=\left.\sum_{i=1}^{m-1} \sum_{j=1}^{v_{i}} \gamma_{j, i}\left[I^{k_{j, i}} x\right]\right|_{\eta_{i-1}} ^{\eta_{i}},
\end{array}\right.
$$

where ${ }^{c} D^{q}$ denotes the Caputo fractional derivative of order $q, f$ is a given continuous function, $\left[I^{k_{j, i}} x\right] \mid \eta_{\eta_{i-1}}^{\eta_{i}}=\left[I^{k_{j, i}} x\right]\left(\eta_{i}\right)-\left[I^{k_{j, i}} x\right]\left(\eta_{i-1}\right), I^{k_{j, i}}$ is the Riemann-Liouville fractional integral of order $k_{j, i}>0, j=1,2, \ldots, v_{i}, v_{i} \in \mathbb{N}=\{1,2, \ldots\}, i=1,2, \ldots, m-1,0=\eta_{0}<\eta_{1}<\eta_{2}<$ $\cdots<\eta_{m-2}<\eta_{m-1}=T$, and $\gamma_{j, i} \in \mathbb{R}$ is such that

$$
\left(T^{n-1}-\sum_{i=1}^{m-1} \sum_{j=1}^{v_{i}} \gamma_{j, i} \frac{\left(\eta_{i}^{k_{j, i}+n-1}-\eta_{i-1}^{k_{j, i}+n-1}\right) \Gamma(n)}{\Gamma\left(k_{j, i}+n\right)}\right) \neq 0
$$

Here we emphasize that Riemann-Liouville type integral boundary conditions involving two indices give rise to a more general situation in contrast to the case of a single index [22]. Furthermore, the present work dealing with an arbitrary-order problem generalizes the results for the problem of order $q \in(1,2]$ obtained in [26]. Several examples are considered to show the worth of the results established in this paper.

We develop some existence results for problem (1.1) by using standard techniques of fixed point theory. The paper is organized as follows. In Section 2 we recall some preliminary facts that we need in the sequel, and Section 3 contains the main results. Section 4 provides some examples for the illustration of the main results.

\section{Preliminaries from fractional calculus}

Let us recall some basic definitions of fractional calculus [2-4].

Definition 2.1 For an at least $n$-times continuously differentiable function $g:[0, \infty) \rightarrow \mathbb{R}$, the Caputo derivative of fractional order $q$ is defined as

$$
{ }^{c} D^{q} g(t)=\frac{1}{\Gamma(n-q)} \int_{0}^{t}(t-s)^{n-q-1} g^{(n)}(s) d s, \quad n-1<q<n, n=[q]+1,
$$

where $[q]$ denotes the integer part of the real number $q$.

Definition 2.2 The Riemann-Liouville fractional integral of order $q$ is defined as

$$
I^{q} g(t)=\frac{1}{\Gamma(q)} \int_{0}^{t} \frac{g(s)}{(t-s)^{1-q}} d s, \quad q>0
$$

provided the integral exists.

Lemma 2.3 For $y \in C[0, T]$, the fractional boundary value problem

$$
\left\{\begin{array}{l}
{ }^{c} D^{q} x(t)=y(t), \quad t \in[0, T], q \in(n-1, n], \\
x(0)=0, x^{\prime}(0)=0, \ldots, x^{(n-2)}=0, \quad x(T)=\left.\sum_{i=1}^{m-1} \sum_{j=1}^{v_{i}} \gamma_{j, i}\left[I^{k_{j, i}} x\right]\right|_{\eta_{i-1}} ^{\eta_{i}}
\end{array}\right.
$$


has a unique solution given by

$$
\begin{aligned}
x(t)= & \frac{1}{\Gamma(q)} \int_{0}^{t}(t-s)^{q-1} y(s) d s-\frac{t^{n-1}}{\delta \Gamma(q)} \int_{0}^{T}(T-s)^{q-1} y(s) d s \\
& +\frac{t^{n-1}}{\delta \Gamma(q)} \sum_{i=1}^{m-1} \sum_{j=1}^{v_{i}} \frac{\gamma_{j, i}}{\Gamma\left(k_{j, i}\right)}\left[\int_{0}^{\eta_{i}} \int_{0}^{s}\left(\eta_{i}-s\right)^{k_{j, i}-1}(s-u)^{q-1} y(u) d u d s\right. \\
& \left.-\int_{0}^{\eta_{i-1}} \int_{0}^{s}\left(\eta_{i-1}-s\right)^{k_{j, i}-1}(s-u)^{q-1} y(u) d u d s\right],
\end{aligned}
$$

where

$$
\delta=\left(T^{n-1}-\sum_{i=1}^{m-1} \sum_{j=1}^{v_{i}} \gamma_{j, i} \frac{\left(\eta_{i}^{k_{j, i}+n-1}-\eta_{i-1}^{k_{j, i}+n-1}\right) \Gamma(n)}{\Gamma\left(k_{j, i}+n\right)}\right) \neq 0 .
$$

Proof The general solution of fractional differential equations in (2.1) can be written as [4]

$$
x(t)=\frac{1}{\Gamma(q)} \int_{0}^{t}(t-s)^{q-1} y(s) d s-c_{0}-c_{1} t-\cdots-c_{n-1} t^{n-1} .
$$

Using the given boundary conditions, it is found that $c_{0}=0, c_{1}=0, \ldots, c_{n-2}=0$. Applying the Riemann-Liouville integral operator $I^{k_{j, i}}$ on (2.4), we get

$$
\begin{aligned}
I^{k_{j, i}} x(t) & =\frac{1}{\Gamma\left(k_{j, i}\right)} \int_{0}^{t}(t-s)^{k_{j, i}-1}\left(\frac{1}{\Gamma(q)} \int_{0}^{s}(s-u)^{q-1} y(u) d u-c_{n-1} s^{n-1}\right) d s \\
= & \frac{1}{\Gamma\left(k_{j, i}\right) \Gamma(q)} \int_{0}^{t} \int_{0}^{s}(t-s)^{k_{j, i}-1}(s-u)^{q-1} y(u) d u d s \\
& -c_{n-1} \frac{1}{\Gamma\left(k_{j, i}\right)} \int_{0}^{t}(t-s)^{k_{j, i}-1} s^{n-1} d s .
\end{aligned}
$$

Using the concept of beta function, we find that

$$
\frac{1}{\Gamma\left(k_{j, i}\right)} \int_{0}^{t}(t-s)^{k_{j, i}-1} s^{n-1} d s=\frac{t^{k_{j, i}+n-1} \Gamma(n)}{\Gamma\left(k_{j, i}+n\right)} .
$$

Now using the condition

$$
x(T)=\left.\sum_{i=1}^{m-1} \sum_{j=1}^{\nu_{i}} \gamma_{j, i}\left[I^{k_{j, i}} x\right]\right|_{\eta_{i-1}} ^{\eta_{i}}=\left[I^{k_{j, i}} x\right]\left(\eta_{i}\right)-\left[I^{k_{j, i}} x\right]\left(\eta_{i-1}\right),
$$

we obtain

$$
\begin{aligned}
& \frac{1}{\Gamma(q)} \int_{0}^{T}(T-s)^{q-1} y(s) d s-c_{n-1} T^{n-1} \\
& \quad=\sum_{i=1}^{m-1} \sum_{j=1}^{v_{i}} \frac{\gamma_{j, i}}{\Gamma(q) \Gamma\left(k_{j, i}\right)}\left[\int_{0}^{\eta_{i}} \int_{0}^{s}\left(\eta_{i}-s\right)^{k_{j, i}-1}(s-u)^{q-1} y(u) d u d s\right.
\end{aligned}
$$




$$
\begin{aligned}
& \left.-\int_{0}^{\eta_{i-1}} \int_{0}^{s}\left(\eta_{i-1}-s\right)^{k_{j, i}-1}(s-u)^{q-1} y(u) d u d s\right] \\
& -c_{n-1} \sum_{i=1}^{m-1} \sum_{j=1}^{v_{i}} \gamma_{j, i} \frac{\left(\eta_{i}^{k_{j, i}+n-1}-\eta_{i-1}^{k_{j, i}+n-1}\right) \Gamma(n)}{\Gamma\left(k_{j, i}+n\right)}
\end{aligned}
$$

which yields

$$
\begin{aligned}
c_{n-1}= & \frac{1}{\delta \Gamma(q)} \int_{0}^{T}(T-s)^{q-1} y(s) d s \\
& -\frac{1}{\delta \Gamma(q)} \sum_{i=1}^{m-1} \sum_{j=1}^{v_{i}} \frac{\gamma_{j, i}}{\Gamma\left(k_{j, i}\right)}\left[\int_{0}^{\eta_{i}} \int_{0}^{s}\left(\eta_{i}-s\right)^{k_{j, i}-1}(s-u)^{q-1} y(u) d u d s\right. \\
& \left.-\int_{0}^{\eta_{i-1}} \int_{0}^{s}\left(\eta_{i-1}-s\right)^{k_{j, i}-1}(s-u)^{q-1} y(u) d u d s\right],
\end{aligned}
$$

where $\delta$ is given by (2.3). Substituting the values of $c_{0}, c_{1}, \ldots, c_{n-2}, c_{n-1}$ in (2.4), we obtain (2.2). This completes the proof.

\section{Main results}

Let $\mathcal{C}:=C([0, T], \mathbb{R})$ denote the Banach space of all continuous functions defined on $[0, T] \times \mathbb{R}$ endowed with a topology of uniform convergence with the norm $\|x\|=$ $\sup _{t \in[0, T]}|x(t)|$.

To prove the existence results for problem (1.1), we need the following known results.

Theorem 3.1 (Leray-Schauder alternative [27, p.4]) Let X be a Banach space. Assume that $T: X \rightarrow X$ is a completely continuous operator and the set

$$
V=\{u \in X \mid u=\mu T u, 0<\mu<1\}
$$

is bounded. Then $T$ has a fixed point in $X$.

Theorem 3.2 [28] Let $X$ be a Banach space. Assume that $\Omega$ is an open bounded subset of $X$ with $\theta \in \Omega$, and let $T: \bar{\Omega} \rightarrow X$ be a completely continuous operator such that

$$
\|T u\| \leq\|u\|, \quad \forall u \in \partial \Omega .
$$

Then $T$ has a fixed point in $\bar{\Omega}$.

By Lemma 2.3, we define an operator $\mathcal{P}: \mathcal{C} \rightarrow \mathcal{C}$ as

$$
\begin{aligned}
(\mathcal{P} x)(t)= & \frac{1}{\Gamma(q)} \int_{0}^{t}(t-s)^{q-1} f(s, x(s)) d s-\frac{t^{n-1}}{\delta \Gamma(q)} \int_{0}^{T}(T-s)^{q-1} f(s, x(s)) d s \\
& +\frac{t^{n-1}}{\delta \Gamma(q)} \sum_{i=1}^{m-1} \sum_{j=1}^{v_{i}} \frac{\gamma_{j, i}}{\Gamma\left(k_{j, i}\right)}\left[\int_{0}^{\eta_{i}} \int_{0}^{s}\left(\eta_{i}-s\right)^{k_{j, i}-1}(s-u)^{q-1} f(u, x(u)) d u d s\right. \\
& \left.-\int_{0}^{\eta_{i-1}} \int_{0}^{s}\left(\eta_{i-1}-s\right)^{k_{j, i}-1}(s-u)^{q-1} f(u, x(u)) d u d s\right], \quad t \in[0, T] .
\end{aligned}
$$


Observe that problem (1.1) has a solution if and only if the associated fixed point problem $\mathcal{P} x=x$ has a fixed point.

For the sake of convenience, we set

$$
\vartheta=\frac{T^{q}}{\Gamma(q+1)}+\frac{T^{q+n-1}}{|\delta| \Gamma(q+1)}+\frac{T^{n-1}}{|\delta|} \sum_{i=1}^{m-1} \sum_{j=1}^{v_{i}}\left|\gamma_{j, i}\right| \frac{\eta_{i}^{q+k_{j, i}}-\eta_{i-1}^{q+k_{j, i}}}{\Gamma\left(q+k_{j, i}+1\right)} .
$$

Theorem 3.3 Assume that there exists a positive constant $L_{1}$ such that $|f(t, x)| \leq L_{1}$ for $t \in[0, T], x \in \mathbb{R}$. Then problem (1.1) has at least one solution.

Proof First of all, we show that the operator $\mathcal{P}$ is completely continuous. Note that the operator $\mathcal{P}$ is continuous in view of the continuity of $f$. Let $\mathcal{B} \subset \mathcal{C}$ be a bounded set. By the assumption that $|f(t, x)| \leq L_{1}$, for $x \in \mathcal{B}$, we have

$$
\begin{aligned}
|(\mathcal{P} x)(t)| \leq & \frac{1}{\Gamma(q)} \int_{0}^{t}(t-s)^{q-1}|f(s, x(s))| d s \\
& +\frac{t^{n-1}}{|\delta| \Gamma(q)} \int_{0}^{T}(T-s)^{q-1}|f(s, x(s))| d s \\
& +\frac{t^{n-1}}{|\delta| \Gamma(q)} \sum_{i=1}^{m-1} \sum_{j=1}^{v_{i}} \frac{\left|\gamma_{j, i}\right|}{\Gamma\left(k_{j, i}\right)}\left(\int_{0}^{\eta_{i}} \int_{0}^{s}\left(\eta_{i}-s\right)^{k_{j, i}-1}(s-u)^{q-1}|f(u, x(u))| d u d s\right. \\
& \left.-\int_{0}^{\eta_{i-1}} \int_{0}^{s}\left(\eta_{i-1}-s\right)^{k_{j, i}-1}(s-u)^{q-1}|f(u, x(u))| d u d s\right) \\
\leq & L_{1}\left[\frac{1}{\Gamma(q)} \int_{0}^{t}(t-s)^{q-1} d s+\frac{t^{n-1}}{|\delta| \Gamma(q)} \int_{0}^{T}(T-s)^{q-1} d s\right. \\
& +\frac{t^{n-1}}{|\delta| \Gamma(q)} \sum_{i=1}^{m-1} \sum_{j=1}^{v_{i}} \frac{\left|\gamma_{j, i}\right|}{\Gamma\left(k_{j, i}\right)}\left(\int_{0}^{\eta_{i}} \int_{0}^{s}\left(\eta_{i}-s\right)^{k_{j, i}-1}(s-u)^{q-1} d u d s\right. \\
& \left.\left.-\int_{0}^{\eta_{i-1}} \int_{0}^{s}\left(\eta_{i-1}-s\right)^{k_{j, i}-1}(s-u)^{q-1} d u d s\right)\right] \\
\leq & L_{1}\left\{\frac{T^{q}}{\Gamma(q+1)}+\frac{T^{q+n-1}}{|\delta| \Gamma(q+1)}+\frac{T^{n-1}}{|\delta|} \sum_{i=1}^{m-1} \sum_{j=1}^{v_{i}}\left|\gamma_{j, i}\right| \frac{\eta_{i}^{q+k_{j, i}}-\eta_{i-1}^{q+k_{j, i}}}{\Gamma\left(q+k_{j, i}+1\right)}\right\}=L_{2},
\end{aligned}
$$

which implies that $\|(\mathcal{P} x)\| \leq L_{2}$. Further, we find that

$$
\begin{aligned}
\left|(\mathcal{P} x)^{\prime}(t)\right| & \\
= & \frac{1}{\Gamma(q-1)} \int_{0}^{t}(t-s)^{q-2}|f(s, x(s))| d s \\
& +\frac{(n-1) t^{n-2}}{|\delta| \Gamma(q)} \int_{0}^{T}(T-s)^{q-1}|f(s, x(s))| d s \\
& +\frac{(n-1) t^{n-2}}{|\delta| \Gamma(q)} \sum_{i=1}^{m-1} \sum_{j=1}^{v_{i}} \frac{\left|\gamma_{j, i}\right|}{\Gamma\left(k_{j, i}\right)}\left(\int_{0}^{\eta_{i}} \int_{0}^{s}\left(\eta_{i}-s\right)^{k_{j, i}-1}(s-u)^{q-1}|f(u, x(u))| d u d s\right. \\
& \left.\quad-\int_{0}^{\eta_{i-1}} \int_{0}^{s}\left(\eta_{i-1}-s\right)^{k_{j, i}-1}(s-u)^{q-1}|f(u, x(u))| d u d s\right)
\end{aligned}
$$




$$
\begin{aligned}
\leq & L_{1}\left[\frac{1}{\Gamma(q-1)} \int_{0}^{t}(t-s)^{q-2} d s+\frac{(n-1) t^{n-2}}{|\delta| \Gamma(q)} \int_{0}^{T}(T-s)^{q-1} d s\right. \\
& +\frac{(n-1) t^{n-2}}{|\delta| \Gamma(q)} \sum_{i=1}^{m-1} \sum_{j=1}^{v_{i}} \frac{\left|\gamma_{j, i}\right|}{\Gamma\left(k_{j, i}\right)}\left(\int_{0}^{\eta_{i}} \int_{0}^{s}\left(\eta_{i}-s\right)^{k_{j, i}-1}(s-u)^{q-1} d u d s\right. \\
& \left.\left.-\int_{0}^{\eta_{i-1}} \int_{0}^{s}\left(\eta_{i-1}-s\right)^{k_{j, i}-1}(s-u)^{q-1} d u d s\right)\right] \\
\leq & L_{1}\left\{\frac{T^{q-1}}{\Gamma(q)}+\frac{(n-1) T^{q+n-2}}{|\delta| \Gamma(q+1)}+\frac{(n-1) T^{n-2}}{|\delta|} \sum_{i=1}^{m-1} \sum_{j=1}^{v_{i}}\left|\gamma_{j, i}\right| \frac{\eta_{i}^{q+k_{j, i}}-\eta_{i-1}^{q+k_{j, i}}}{\Gamma\left(q+k_{j, i}+1\right)}\right\}=L_{3} .
\end{aligned}
$$

Hence, for $t_{1}, t_{2} \in[0, T]$, we have

$$
\left|(\mathcal{P} x)\left(t_{2}\right)-(\mathcal{P} x)\left(t_{1}\right)\right| \leq \int_{t_{1}}^{t_{2}}\left|(\mathcal{P} x)^{\prime}(s)\right| d s \leq L_{3}\left(t_{2}-t_{1}\right) .
$$

This implies that $\mathcal{P}$ is equicontinuous on $[0, T]$. Thus, by the Arzela-Ascoli theorem, the operator $\mathcal{P}: \mathcal{C} \rightarrow \mathcal{C}$ is completely continuous.

Next, we consider the set

$$
V=\{x \in \mathcal{C} \mid x=\mu \mathcal{P} x, 0<\mu<1\}
$$

and show that the set $V$ is bounded. Let $x \in V$, then $x=\mu \mathcal{P} x, 0<\mu<1$. For any $t \in[0, T]$, we have

$$
\begin{aligned}
|x(t)|= & \mu|(\mathcal{P} x)(t)| \\
\leq & \frac{1}{\Gamma(q)} \int_{0}^{t}(t-s)^{q-1}|f(s, x(s))| d s \\
& +\frac{t^{n-1}}{|\delta| \Gamma(q)} \int_{0}^{T}(T-s)^{q-1}|f(s, x(s))| d s \\
& +\frac{t^{n-1}}{|\delta| \Gamma(q)} \sum_{i=1}^{m-1} \sum_{j=1}^{v_{i}} \frac{\left|\gamma_{j, i}\right|}{\Gamma\left(k_{j, i}\right)}\left(\int_{0}^{\eta_{i}} \int_{0}^{s}\left(\eta_{i}-s\right)^{k_{j, i}-1}(s-u)^{q-1}|f(u, x(u))| d u d s\right. \\
& \left.-\int_{0}^{\eta_{i-1}} \int_{0}^{s}\left(\eta_{i-1}-s\right)^{k_{j, i}-1}(s-u)^{q-1}|f(u, x(u))| d u d s\right) \\
\leq & L_{1}\left\{\frac{T^{q}}{\Gamma(q+1)}+\frac{T^{q+n-1}}{|\delta| \Gamma(q+1)}+\frac{T^{n-1}}{|\delta|} \sum_{i=1}^{m-1} \sum_{j=1}^{v_{i}}\left|\gamma_{j, i}\right| \frac{\eta_{i}^{q+k_{j, i}}-\eta_{i-1}^{q+k_{j, i}}}{\Gamma\left(q+k_{j, i}+1\right)}\right\}=M_{1} .
\end{aligned}
$$

Thus, $\|x\| \leq M_{1}$ for any $t \in[0, T]$. So, the set $V$ is bounded. Thus, by the conclusion of Theorem 3.1, the operator $\mathcal{P}$ has at least one fixed point, which implies that (1.1) has at least one solution.

Theorem 3.4 Let there exist a small positive number $\tau$ such that $|f(t, x)| \leq \epsilon|x|$ for $0<|x|<\tau$, with $0<\epsilon \leq 1 / \vartheta$, where $\vartheta$ is given by (3.2). Then problem (1.1) has at least one solution. 
Proof Let us define $\mathcal{B}_{\tau}=\{x \in \mathcal{C} \mid\|x\|<\tau\}$ and take $x \in \mathcal{C}$ such that $\|x\|=\tau$, that is, $x \in \partial \mathcal{B}_{\tau}$. As before, it can be shown that $\mathcal{P}$ is completely continuous and

$$
\begin{aligned}
&\|\mathcal{P} x\| \leq \sup _{t \in[0, t]}\left\{\frac{1}{\Gamma(q)} \int_{0}^{t}(t-s)^{q-1}|f(s, x(s))| d s+\frac{t^{n-1}}{|\delta| \Gamma(q)} \int_{0}^{T}(T-s)^{q-1}|f(s, x(s))| d s\right. \\
&+\frac{t^{n-1}}{|\delta| \Gamma(q)} \sum_{i=1}^{m-1} \sum_{j=1}^{v_{i}} \frac{\left|\gamma_{j, i}\right|}{\Gamma\left(k_{j, i}\right)}\left(\int_{0}^{\eta_{i}} \int_{0}^{s}\left(\eta_{i}-s\right)^{k_{j, i}-1}(s-u)^{q-1}|f(u, x(u))| d u d s\right. \\
&\left.\left.-\int_{0}^{\eta_{i-1}} \int_{0}^{s}\left(\eta_{i-1}-s\right)^{k_{j, i}-1}(s-u)^{q-1}|f(u, x(u))| d u d s\right)\right\} \\
& \leq \vartheta \epsilon\|x\|,
\end{aligned}
$$

which, in view of the given condition $(\epsilon \vartheta \leq 1)$, gives $\|\mathcal{P} x\| \leq\|x\|, x \in \partial \mathcal{B}_{\tau}$. Therefore, by Theorem 3.2, the operator $\mathcal{P}$ has at least one fixed point, which in turn implies that problem (1.1) has at least one solution.

Our next result is based on Leray-Schauder nonlinear alternative.

Lemma 3.5 (Nonlinear alternative for single-valued maps [27, p.135]) Let E be a Banach space, $C$ be a closed, convex subset of $E, U$ be an open subset of $C$ and $0 \in U$. Suppose that $F: \bar{U} \rightarrow C$ is a continuous, compact (that is, $F(\bar{U})$ is a relatively compact subset of $C$ ) map. Then either

(i) F has a fixed point in $\bar{U}$, or

(ii) there are $u \in \partial U$ (the boundary of $U$ in $C$ ) and $\lambda \in(0,1)$ with $u=\lambda F(u$ ).

\section{Theorem 3.6 Assume that}

$\left(\mathrm{A}_{1}\right)$ there exist a function $\sigma \in C\left([0,1], \mathbb{R}^{+}\right)$and a nondecreasing function $\psi: \mathbb{R}^{+} \rightarrow \mathbb{R}^{+}$such that $|f(t, x)| \leq \sigma(t) \psi(\|x\|), \forall(t, x) \in[0, T] \times \mathbb{R} ;$

$\left(\mathrm{A}_{2}\right)$ there exists a constant $M>0$ such that

$$
\frac{M}{\psi(M) \vartheta\|\sigma\|}>1
$$

Then boundary value problem (1.1) has at least one solution on $[0, T]$.

Proof Consider the operator $\mathcal{P}: \mathcal{C} \rightarrow \mathcal{C}$ defined by (3.1). We show that $\mathcal{P}$ maps bounded sets into bounded sets in $C([0, T], \mathbb{R})$. For a positive number $r$, let $B_{r}=\{x \in C([0, T], \mathbb{R})$ : $\|x\| \leq r\}$ be a bounded set in $C([0, T], \mathbb{R})$. Then

$$
\begin{aligned}
\|\mathcal{P} x\| \leq & \sup _{t \in[0, T]}\left\{\frac{1}{\Gamma(q)} \int_{0}^{t}(t-s)^{q-1}|f(s, x(s))| d s\right. \\
& +\frac{t^{n-1}}{|\delta| \Gamma(q)} \int_{0}^{T}(T-s)^{q-1}|f(s, x(s))| d s \\
& +\frac{t^{n-1}}{|\delta| \Gamma(q)} \sum_{i=1}^{m-1} \sum_{j=1}^{v_{i}} \frac{\left|\gamma_{j, i}\right|}{\Gamma\left(k_{j, i}\right)}\left(\int_{0}^{\eta_{i}} \int_{0}^{s}\left(\eta_{i}-s\right)^{k_{j, i}-1}(s-u)^{q-1}|f(u, x(u))| d u d s\right.
\end{aligned}
$$




$$
\begin{aligned}
& \left.\left.-\int_{0}^{\eta_{i-1}} \int_{0}^{s}\left(\eta_{i-1}-s\right)^{k_{j, i}-1}(s-u)^{q-1}|f(u, x(u))| d u d s\right)\right\} \\
\leq & \psi(r)\left\{\frac{T^{q}}{\Gamma(q+1)}+\frac{T^{q+n-1}}{|\delta| \Gamma(q+1)}+\frac{T^{n-1}}{|\delta|} \sum_{i=1}^{m-1} \sum_{j=1}^{\nu_{i}}\left|\gamma_{j, i}\right| \frac{\eta_{i}^{q+k_{j, i}}-\eta_{i-1}^{q+k_{j, i}}}{\Gamma\left(q+k_{j, i}+1\right)}\right\}\|\sigma\| .
\end{aligned}
$$

Next we show that $F$ maps bounded sets into equicontinuous sets of $C([0,1], \mathbb{R})$. Let $t^{\prime}, t^{\prime \prime} \in$ $[0,1]$ with $t^{\prime}<t^{\prime \prime}$ and $x \in B_{r}$, where $B_{r}$ is a bounded set of $C([0,1], \mathbb{R})$. Then we obtain

$$
\begin{aligned}
\mid(\mathcal{P} x) & \left(t^{\prime \prime}\right)-(\mathcal{P} x)\left(t^{\prime}\right) \mid \\
= & \mid \frac{1}{\Gamma(q)} \int_{0}^{t^{\prime \prime}}\left(t^{\prime \prime}-s\right)^{q-1} f(s, x(s)) d s-\frac{1}{\Gamma(q)} \int_{0}^{t^{\prime}}\left(t^{\prime}-s\right)^{q-1} f(s, x(s)) d s \\
& -\frac{\left[\left(t^{\prime \prime}\right)^{n-1}-\left(t^{\prime}\right)^{n-1}\right]}{\delta \Gamma(q)} \int_{0}^{T}(T-s)^{q-1} f(s, x(s)) d s \\
& +\frac{\left[\left(t^{\prime \prime}\right)^{n-1}-\left(t^{\prime \prime}\right)^{n-1}\right]}{\delta \Gamma(q)} \sum_{i=1}^{m-1} \sum_{j=1}^{v_{i}} \frac{\gamma_{j, i}}{\Gamma\left(k_{j, i}\right)}\left[\int_{0}^{\eta_{i}} \int_{0}^{s}\left(\eta_{i}-s\right)^{k_{j, i}-1}(s-u)^{q-1} f(u, x(u)) d u d s\right. \\
& \left.-\int_{0}^{\eta_{i-1}} \int_{0}^{s}\left(\eta_{i-1}-s\right)^{k_{j, i}-1}(s-u)^{q-1} f(u, x(u)) d u d s\right] \mid \\
\leq & \frac{1}{\Gamma(q)} \int_{0}^{t^{\prime}}\left|\left(t^{\prime \prime}-s\right)^{q-1}-\left(t^{\prime}-s\right)^{q-1}\right| \psi(r) \sigma(s) d s+\frac{1}{\Gamma(q)} \int_{t^{\prime}}^{t^{\prime \prime}}\left|t^{\prime \prime}-s\right|^{q-1} \psi(r) \sigma(s) d s \\
& +\frac{\left|\left(t^{\prime \prime}\right)^{n-1}-\left(t^{\prime}\right)^{n-1}\right|}{|\delta| \Gamma(q)} \int_{0}^{T}|T-s|^{q-1} \psi(r) \sigma(s) d s \\
& +\frac{\left|\left(t^{\prime \prime}\right)^{n-1}-\left(t^{\prime}\right)^{n-1}\right|}{|\delta| \Gamma(q)} \sum_{i=1}^{m-1} \sum_{j=1}^{v_{i}} \frac{\left|\gamma_{j, i}\right|}{\Gamma\left(k_{j, i}\right)}\left[\int_{0}^{\eta_{i}} \int_{0}^{s}\left|\left(\eta_{i}-s\right)^{k_{j, i}-1}(s-u)^{q-1}\right| \psi(r) \sigma(u) d u d s\right. \\
& \left.+\int_{0}^{\eta_{i-1}} \int_{0}^{s}\left|\left(\eta_{i-1}-s\right)^{k_{j, i}-1}(s-u)^{q-1}\right| \psi(r) \sigma(u) d u d s\right] .
\end{aligned}
$$

Obviously the right-hand side of the above inequality tends to zero independently of $x \in B_{r}$ as $t^{\prime \prime}-t^{\prime} \rightarrow 0$. As $\mathcal{P}: C([0, T], \mathbb{R}) \rightarrow C([0, T], \mathbb{R})$ satisfies the above assumptions, therefore it follows by the Arzelá-Ascoli theorem that $\mathcal{P}$ is completely continuous.

Let $x$ be a solution. Then, for $t \in[0, T]$, and following the similar computations as before, we find that

$$
\begin{aligned}
|x(t)| & =|\mu(\mathcal{P} x)(t)| \\
& \leq \psi(\|x\|)\left\{\frac{T^{q}}{\Gamma(q+1)}+\frac{T^{q+n-1}}{|\delta| \Gamma(q+1)}+\frac{T^{n-1}}{|\delta|} \sum_{i=1}^{m-1} \sum_{j=1}^{v_{i}}\left|\gamma_{j, i}\right| \frac{\eta_{i}^{q+k_{j, i}}-\eta_{i-1}^{q+k_{j, i}}}{\Gamma\left(q+k_{j, i}+1\right)}\right\}\|\sigma\| \\
& =\psi(\|x\|) \vartheta\|\sigma\| .
\end{aligned}
$$

In consequence, we have

$$
\frac{\|x\|}{\psi(\|x\|) \vartheta\|\sigma\|} \leq 1
$$


Thus, by $\left(\mathrm{A}_{2}\right)$, there exists $M$ such that $\|x\| \neq M$. Let us set

$$
V=\{x \in C([0, T], \mathbb{R}):\|x\|<M+1\}
$$

Note that the operator $\mathcal{P}: \bar{V} \rightarrow C([0, T], \mathbb{R})$ is continuous and completely continuous. From the choice of $V$, there is no $x \in \partial V$ such that $x=\mu \mathcal{P}(x)$ for some $\mu \in(0,1)$. Consequently, by the nonlinear alternative of Leray-Schauder type (Lemma 3.5), we deduce that $\mathcal{P}$ has a fixed point $x \in \bar{V}$ which is a solution of problem (1.1). This completes the proof.

Finally we prove an existence and uniqueness result by means of Banach's contraction mapping principle.

Theorem 3.7 Suppose that $f:[0, T] \times \mathbb{R} \rightarrow \mathbb{R}$ is a continuous function and satisfies the following assumption:

$\left(\mathrm{A}_{3}\right)|f(t, x)-f(t, y)| \leq L|x-y|, \forall t \in[0,1], L>0, x, y \in \mathbb{R}$.

Then boundary value problem (1.1) has a unique solution provided

$$
L<1 / \vartheta
$$

where $\vartheta$ is given by (3.2).

Proof With $r \geq M \vartheta /(1-L \vartheta)$, we define $B_{r}=\{x \in \mathcal{C}:\|x\| \leq r\}$, where $M=\sup _{t \in[0, T]} \mid f(t$, $0) \mid<\infty$ and $\vartheta$ is given by (2.3). Then we show that $\mathcal{P} B_{r} \subset B_{r}$. For $x \in B_{r}$, by means of the inequality $|f(s, x(s))| \leq|f(s, x(s))-f(s, 0)|+|f(s, 0)| \leq L\|x\|+M \leq L r+M$, it can easily be shown that

$$
\|\mathcal{P} x\|=(L r+M) \vartheta \leq r .
$$

Now, for $x, y \in \mathcal{C}$ and for each $t \in[0, T]$, we obtain

$$
\begin{aligned}
& \|(\mathcal{P} x)-(\mathcal{P} y)\| \\
& \leq \sup _{t \in[0, T]}\left\{\frac{1}{\Gamma(q)} \int_{0}^{t}(t-s)^{q-1}|f(s, x(s))-f(s, y(s))| d s\right. \\
& \quad+\frac{t^{n-1}}{\delta \Gamma(q)} \int_{0}^{T}(T-s)^{q-1}|f(s, x(s))-f(s, y(s))| d s \\
& \quad+\frac{t^{n-1}}{\delta \Gamma(q)} \sum_{i=1}^{m-1} \sum_{j=1}^{v_{i}} \frac{\left|\gamma_{j, i}\right|}{\Gamma\left(k_{j, i}\right)}\left[\int_{0}^{\eta_{i}} \int_{0}^{s}\left(\eta_{i}-s\right)^{k_{j, i}-1}(s-u)^{q-1}|f(u, x(u))-f(u, y(u))| d u d s\right. \\
& \left.\left.\quad-\int_{0}^{\eta_{i-1}} \int_{0}^{s}\left(\eta_{i-1}-s\right)^{k_{j, i}-1}(s-u)^{q-1}|f(u, x(u))-f(u, y(u))| d u d s\right]\right\} \\
& \leq L \vartheta\|x-y\| .
\end{aligned}
$$


Note that $\vartheta$ depends only on the parameters involved in the problem. As $L \vartheta<1$, therefore $\mathcal{P}$ is a contraction. Hence, by Banach's contraction mapping principle, problem (1.1) has a unique solution on $[0, T]$.

\section{Examples}

In this section, we present some examples for the illustration of the results established in Section 3 by choosing the nonlinear function $f(t, x(t))$ appropriately. Let us consider the following nonlocal boundary value problem:

$$
\left\{\begin{array}{l}
{ }^{c} D^{11 / 2} x(t)=f(t, x(t)), \quad t \in[0,2], \\
x(0)=0, x^{\prime}(0)=0, \ldots, x^{(4)}(0)=0, \quad x(T)=\left.\sum_{i=1}^{m-1} \sum_{j=1}^{v_{i}} \gamma_{j, i}\left[I^{k_{j, i}} x\right]\right|_{\eta_{i-1}} ^{\eta_{i}},
\end{array}\right.
$$

where $q=11 / 2, n=6, m=5, \eta_{0}=0, \eta_{1}=1 / 2, \eta_{2}=1, \eta_{3}=3 / 2, \eta_{4}=T=2, v_{1}=1, v_{2}=2$, $v_{3}=3, v_{4}=4, \gamma_{1,1}=4, \gamma_{1,2}=7, \gamma_{2,2}=8, \gamma_{1,3}=10, \gamma_{2,3}=15, \gamma_{3,3}=21, \gamma_{1,4}=3, \gamma_{2,4}=9, \gamma_{3,4}=$ $15, \gamma_{4,4}=21, k_{1,1}=3 / 2, k_{1,2}=5 / 4, k_{2,2}=7 / 4, k_{1,3}=1 / 2, k_{2,3}=5 / 4, k_{3,3}=9 / 4, k_{1,4}=3 / 4$, $k_{2,4}=7 / 4, k_{3,4}=11 / 4, k_{4,4}=15 / 4$. Using the given data, we find that

$$
\delta=T^{n-1}-\sum_{i=1}^{m-1} \sum_{j=1}^{v_{i}} \gamma_{j, i} \frac{\left(\eta_{i}^{k_{j, i}+n-1}-\eta_{i-1}^{k_{j, i}+n-1}\right) \Gamma(n)}{\Gamma\left(k_{j, i}+n\right)} \simeq-113.395760,
$$

and

$$
\vartheta=\frac{T^{q}}{\Gamma(q+1)}+\frac{T^{q+n-1}}{|\delta| \Gamma(q+1)}+\frac{T^{n-1}}{|\delta|} \sum_{i=1}^{m-1} \sum_{j=1}^{v_{i}}\left|\gamma_{j, i}\right| \frac{\eta_{i}^{q+k_{j, i}}-\eta_{i-1}^{q+k_{j, i}}}{\Gamma\left(q+k_{j, i}+1\right)} \simeq 0.37757 .
$$

(a) As a first example, let us take

$$
f(t, x(t))=\frac{e^{\left(2-\cos ^{2} x(t)\right)^{2}}\left[5 \sin 2 t+10 \ln \left(1+4 \cos ^{2} x(t)\right)\right]}{\sqrt{(26+\sin x(t))}} .
$$

Observe that $|f(t, x)| \leq L_{1}$ with $L_{1}=e^{4}(1+2 \ln 5)$. Thus the hypothesis of Theorem 3.3 is satisfied. Hence, by the conclusion of Theorem 3.3, problem (4.1) with $f(t, x(t))$ given by (4.2) has at least one solution.

(b) Let us consider

$$
f(t, x(t))=x\left(b^{4}+x^{4}(t)\right)^{\frac{1}{4}}+2\left(1+\sin \left(t^{4}+1\right)\right)^{3}(1-\cos x(t)), \quad x \neq 0, b>0 .
$$

For sufficiently small $x$ (ignoring $x^{2}$ and higher powers of $x$ ), we have

$$
\left|x\left(b^{4}+x^{4}(t)\right)^{\frac{1}{4}}+2\left(1+\sin \left(t^{4}+1\right)\right)^{3}(1-\cos x(t))\right| \leq b|x| .
$$

Choosing $b \leq 1 / \vartheta$, all the assumptions of Theorem 3.4 hold. Therefore, the conclusion of Theorem 3.4 implies that problem (4.1) with $f(t, x(t))$ given by (4.3) has at least one solution.

(c) Consider

$$
f(t, x(t))=\sqrt{t+14}\left(1+\frac{|x|}{1+|x|}\right) \leq \sigma(t) \psi(\|x\|)
$$


with $\sigma(t)=\sqrt{t+14}$ and $\psi(\|x\|)=2$. Using $\|\sigma\|=4, \vartheta \simeq 0.37757$, we find by condition $\left(\mathrm{A}_{2}\right)$ that $M>M_{1}$, where $M_{1} \simeq 3.02056$. Thus all the assumptions of Theorem 3.6 are satisfied. Hence, it follows by Theorem 3.6 that problem (4.1) with $f(t, x(t))$ defined by (4.4) has at least one solution.

(d) For the illustration of the existence-uniqueness result, we choose

$$
f(t, x(t))=\frac{1}{\sqrt{(t+16)}}\left(\frac{|x|}{1+|x|}+\tan ^{-1} x\right)+\sqrt{4+\sin t} .
$$

Clearly, $L=1 / 2$ as $|f(t, x)-f(t, y)| \leq \frac{1}{2}|x-y|$ and $L<\frac{1}{\vartheta} \simeq 2.64852$. Therefore all the conditions of Theorem 3.7 hold, and consequently there exists a unique solution for problem (4.1) with $f(t, x(t))$ given by (4.5).

\section{Competing interests}

The authors declare that they have no competing interests.

\section{Authors' contributions}

Each of the authors, AA, SKN, RPA and BA, contributed to each part of this work equally and read and approved the final version of the manuscript.

\section{Author details}

${ }^{1}$ Department of Mathematics, Faculty of Science, King Abdulaziz University, P.O. Box 80203, Jeddah, 21589, Saudi Arabia. ${ }^{2}$ Department of Mathematics, University of loannina, loannina, 451 10, Greece. ${ }^{3}$ Department of Mathematics, Texas A\&M University, Kingsville, 78363-8202, USA.

\section{Acknowledgements}

This research was partially supported by the Deanship of Scientific Research (DSR), King Abdulaziz University, Jeddah, Saudi Arabia.

Received: 6 November 2013 Accepted: 1 December 2013 Published: 13 Dec 2013

\section{References}

1. Miller, KS, Ross, B: An Introduction to the Fractional Calculus and Differential Equations. Wiley, New York (1993)

2. Samko, SG, Kilbas, AA, Marichev, OI: Fractional Integrals and Derivatives, Theory and Applications. Gordon \& Breach, Yverdon (1993)

3. Podlubny, I: Fractional Differential Equations. Academic Press, San Diego (1999)

4. Kilbas, AA, Srivastava, HM, Trujillo, JJ: Theory and Applications of Fractional Differential Equations. North-Holland Mathematics Studies, vol. 204. Elsevier, Amsterdam (2006)

5. Lakshmikantham, V, Leela, S, Vasundhara Devi, J: Theory of Fractional Dynamic Systems. Cambridge Academic Publishers, Cambridge (2009)

6. Baleanu, D, Diethelm, K, Scalas, E, Trujillo, JJ: Fractional Calculus: Models and Numerical Methods. Series on Complexity, Nonlinearity and Chaos. World Scientific, Boston (2012)

7. Benchohra, M, Hamani, S, Ntouyas, SK: Boundary value problems for differential equations with fractional order and nonlocal conditions. Nonlinear Anal. 71, 2391-2396 (2009)

8. Ahmad, B, Nieto, JJ: Existence of solutions for nonlocal boundary value problems of higher-order nonlinear fractional differential equations. Abstr. Appl. Anal. 2009, Article ID 494720 (2009)

9. Agarwal, RP, Benchohra, M, Hamani, S: A survey on existence results for boundary value problems of nonlinear fractional differential equations and inclusions. Acta Appl. Math. 109, 973-1033 (2010)

10. Ahmad, B: Existence of solutions for irregular boundary value problems of nonlinear fractional differential equations. Appl. Math. Lett. 23, 390-394 (2010)

11. Balachandran, $\mathrm{K}$, Trujillo, JJ: The nonlocal Cauchy problem for nonlinear fractional integrodifferential equations in Banach spaces. Nonlinear Anal. 72, 4587-4593 (2010)

12. Baleanu, D, Mustafa, OG: On the global existence of solutions to a class of fractional differential equations. Comput. Math. Appl. 59, 1835-1841 (2010)

13. Zhong, W, Lin, W: Nonlocal and multiple-point boundary value problem for fractional differential equations. Comput. Math. Appl. 39, 1345-1351 (2010)

14. Gafiychuk, V, Datsko, B, Meleshko, V: Mathematical modeling of different types of instabilities in time fractional reaction-diffusion systems. Comput. Math. Appl. 59, 1101-1107 (2010)

15. Ahmad, B, Nieto, JJ: Riemann-Liouville fractional integro-differential equations with fractional nonlocal integral boundary conditions. Bound. Value Probl. 2011, Article ID 36 (2011)

16. Ahmad, B, Ntouyas, SK: A four-point nonlocal integral boundary value problem for fractional differential equations of arbitrary order. Electron. J. Qual. Theory Differ. Equ. 22, 1-15 (2011)

17. Cuevas, C, Soto, H, Sepulveda, A: Almost periodic and pseudo-almost periodic solutions to fractional differential and integrodifferential equations. Appl. Math. Comput. 218, 1735-1745 (2011) 
18. Ford, NJ, Morgado, ML: Fractional boundary value problems: analysis and numerical methods. Fract. Calc. Appl. Anal. 14(4), 554-567 (2011)

19. Ahmad, B, Ntouyas, SK: A note on fractional differential equations with fractional separated boundary conditions. Abstr. Appl. Anal. 2012, Article ID 818703 (2012)

20. Aghajani, A, Jalilian, Y, Trujillo, Jj: On the existence of solutions of fractional integro-differential equations. Fract. Calc. Appl. Anal. 15(2), 44-69 (2012)

21. Ahmad, B, Ntouyas, SK: Nonlinear fractional differential equations and inclusions of arbitrary order and multi-strip boundary conditions. Electron. J. Differ. Equ. 98, 1-22 (2012)

22. Ahmad, B, Ntouyas, SK, Alsaedi, A: A study of nonlinear fractional differential equations of arbitrary order with Riemann-Liouville type multi-strip boundary conditions. Math. Probl. Eng. 2013, Article ID 320415 (2013)

23. Debbouche, A, Baleanu, D, Agarwal, RP: Nonlocal nonlinear integrodifferential equations of fractional orders. Bound. Value Probl. 2012, Article ID 78 (2012)

24. Nyamoradi, N, Baleanu, D, Agarwal, RP: On a multipoint boundary value problem for a fractional order differential inclusion on an infinite interval. Adv. Math. Phys. 2013, Article ID 823961 (2013)

25. Baleanu, D, Agarwal, RP, Mohammadi, H, Rezapour, S: Some existence results for a nonlinear fractional differential equation on partially ordered Banach spaces. Bound. Value Probl. 2013, Article ID 112 (2013)

26. Sudsutad, W, Tariboon, J: Existence results of fractional integro-differential equations with $m$-point multi-term fractional order integral boundary conditions. Bound. Value Probl. 2012, Article ID 94 (2012)

27. Granas, A, Dugundji, J: Fixed Point Theory. Springer, New York (2005)

28. Smart, DR: Fixed Point Theorems. Cambridge University Press, Cambridge (1980)

10.1186/1687-1847-2013-369

Cite this article as: Alsaedi et al.: A nonlocal multi-point multi-term fractional boundary value problem with Riemann-Liouville type integral boundary conditions involving two indices. Advances in Difference Equations 2013, 2013:369

\section{Submit your manuscript to a SpringerOpen ${ }^{\circ}$ journal and benefit from:}

- Convenient online submission

- Rigorous peer review

Immediate publication on acceptance

Open access: articles freely available online

- High visibility within the field

- Retaining the copyright to your article 